\title{
Studying How Individuals Who Express the Feeling of Loneliness in an Online Loneliness Forum Communicate in a Nonloneliness Forum: Observational Study
}

\author{
Anietie Andy, $\mathrm{PhD}$ \\ Penn Medicine, Philadelphia, PA, United States
}

\section{Corresponding Author:}

Anietie Andy, $\mathrm{PhD}$

Penn Medicine

3400 Civic Blvd

Philadelphia, PA,

United States

Phone: 12024864095

Email: andyanietie@gmail.com

\begin{abstract}
Background: Loneliness is a public health concern, and increasingly, individuals experiencing loneliness are seeking support on online forums, some of which focus on discussions around loneliness (loneliness forums). Some of these individuals may also seek support around loneliness on online forums not related to loneliness or well-being (nonloneliness forums). Hence, to design and implement appropriate and efficient online loneliness interventions, it is important to understand how individuals who express and seek support around loneliness on online loneliness forums communicate in nonloneliness forums; this could provide further insights into the support needs and concerns of these users.
\end{abstract}

Objective: This study aims to explore how users who express the feeling of loneliness and seek support around loneliness on an online loneliness forum communicate in an online nonloneliness forum.

Methods: A total of 2401 users who expressed loneliness in posts published on a loneliness forum on Reddit and had published posts in a nonloneliness forum were identified. Using latent Dirichlet allocation (a natural language processing algorithm); Linguistic Inquiry and Word Count (a psycholinguistic dictionary); and the word score-based language features valence, arousal, and dominance, the language use differences in posts published in the nonloneliness forum by these users compared to a control group of users who did not belong to any loneliness forum on Reddit were determined.

Results: It was found that in posts published in the nonloneliness forum, users who expressed loneliness tend to use more words associated with the Linguistic Inquiry and Word Count categories on sadness (Cohen $d=0.10$ ) and seeking to socialize (Cohen $d=0.114$ ), and use words associated with valence (Cohen $d=0.364$ ) and dominance (Cohen $d=0.117$ ). In addition, they tend to publish posts related to latent Dirichlet allocation topics such as relationships (Cohen $d=0.105$ ) and family and friends and mental health (Cohen $d=0.10)$.

Conclusions: There are clear distinctions in language use in nonloneliness forum posts by users who express loneliness compared to a control group of users. These findings can help with the design and implementation of online interventions around loneliness.

(JMIR Form Res 2021;5(7):e28738) doi: 10.2196/28738

\section{KEYWORDS}

loneliness; Reddit; nonloneliness; mental health; eHealth; forum; online forum; communication; natural language processing; language; linguistics

\section{Introduction}

Loneliness is a public health challenge [1]; it affects the well-being of individuals of all age groups $[2,3]$ and has been linked to early death $[4,5]$, depression [6,7], and heart disease [8].
Several prior works $[1,9,10]$ have analyzed data from social media platforms and online forums, some of which focus on discussions around loneliness (loneliness forums) to understand the support needs of individuals who express loneliness on these platforms. These individuals may also seek support and express concerns as it relates to loneliness, such as how to develop or 
maintain relationships, on online forums not focused on discussions around loneliness or well-being (nonloneliness forums). To better understand the support needs of users who express loneliness on online loneliness forums and to design and implement appropriate and efficient online interventions, it is important to study how these individuals communicate in nonloneliness forums.

Using natural language processing methods, prior works determined that the language used in posts published on social media platforms and online forums can be used to gain insights into how users communicate on these forums and the types of support they seek and express, as it relates to their health and well-being. For example, language used in posts published on Facebook was used to train a machine learning model to predict patients risk for cardiovascular disease [11]. In addition, language used on online forum posts were used to determine the support needs of users in substance use recovery forums [12], a COVID-19 online forum [13,14], and an online cancer forum [15-17]. Regarding loneliness, prior works analyzed social media data belonging to individuals who expressed loneliness; for example, Guntuku et al [1] analyzed Twitter posts from users who self-declared feeling lonely, and it was determined that the language used by these users in their Twitter posts was more associated with mental health concerns. Kivran-Swaine et al [18] determined that posts in which users expressed loneliness tended to receive more responses compared to other posts by the same users. Ruiz et al [9] showed that the more loneliness users express on social media, the less online relationships they had. Hunt et al [10] showed that there is an association between less social media use and a decrease in the feeling of loneliness and depression.

Similar to prior works, in this study, using natural language processing methods, language used on posts published on a nonloneliness online forum are analyzed to gain insights into the support needs and concerns of individuals who express loneliness in a loneliness online forum. Potentially, information gleaned from the analyses in this study will provide further insights into the support needs and concerns of individuals expressing loneliness on online loneliness forums, thereby informing loneliness interventions.

\section{Methods}

\section{Data and Design}

For the analysis in this study, data from Reddit was used. Reddit is made up of more than 1 million subforums (called subreddits) [19] focused on discussions around specific topics such as depression, loneliness, and open-ended questions spanning various topics. In addition, Reddit allows members to join several of these forums; hence, it is possible to get posts published in a nonloneliness forum by users who are members of a loneliness forum. Using Google's BigQuery [20], which is a data warehouse with publicly accessible Reddit data sets published between December 2015 and August 2019, posts from Reddit forums focused on discussions around loneliness were identified by selecting the forums that contained the word "Lonely" in its name (eg, "/r/lonelyheartbeats," "/r/iAMlonely," and "/r/Lonely"). It was observed that the forum $/ r /$ Lonely had more posts and members compared to the other forums, which each had less than 200 published posts during the time period in which the data was collected. Hence, for the analysis in this study, usernames of members of / $/$ /Lonely were used; specifically, the usernames of 9956 users who had published a total of 15,012 posts on the /r/Lonely forum were selected.

To identify the other Reddit forums in which these users belong to and tend to publish posts, using the usernames from $/ r /$ Lonely, all the forums on Reddit were searched to determine the forums in which these $/ r /$ Lonely users tend to publish posts. It was observed that the forums with the most number of these users as members are / $r$ /AskReddit (a forum in which users seek advice and ask open-ended questions on various topics) and Ir/depression (a forum focused on discussions around depression) with posts by $24 \%(n=2401)$ and $20 \%(n=2031)$, respectively, of the $/ r$ Lonely users $(\mathrm{N}=9956)$ in the data set. Since the focus of this study is to determine how individuals who express loneliness on an online loneliness forum communicate in nonloneliness forums (ie, forums not focused on loneliness or well-being), for all the analysis in this study, data from /r/AskReddit by 2401 users who expressed loneliness in $/ r /$ Lonely were used. The author reviewed the posts $(\mathrm{N}=4001)$ published on /r/Lonely by these users and observed that these users expressed feeling lonely in their/r/Lonely posts by stating that they were feeling lonely (eg, rephrased "I am a 25 years old female and I am always lonely"), implied that they were feeling lonely (eg, rephrased "I moved to a new city and I don't know anyone"), or sought support as it relates to loneliness (eg, rephrased "Where can I find tools online to help with loneliness?").

Each of the 2401 users who posted on /r/Lonely and had published posts on /r/AskReddit were matched with a control group user who had no published posts on any loneliness forum on Reddit and had published posts on /r/AskReddit between December 2015 and August 2019.

Table 1 shows information about the data set.

Table 1. Summary of /r/AskReddit posts published between December 2015 and August 2019 by /r/Lonely users and a control group.

\begin{tabular}{lll}
\hline Variables & /r/Lonely users & Control group \\
\hline Users, $\mathrm{n}$ & 2401 & 2401 \\
Posts, $\mathrm{n}$ & 25,834 & 34,718 \\
\hline
\end{tabular}

In this study, the following methods were used to determine language use differences in $/ r / A s k R e d d i t$ posts by users who express loneliness compared to the control group: a topic modeling approach, a dictionary-based approach, and a word score-based approach. Cohen $d$, which indicates the standardized difference between means, was used to report the effect sizes. In this study, only results with Cohen $d$ greater than 
or equal to a threshold (ie, 0.10) and that are significant at Bonferroni-corrected $P$ values <.001 are reported.

The topic modeling approach and the dictionary-based approaches were used because prior works used these approaches to gain insights from social media data about the language use differences between individuals in different genders [21] and age groups [22], and to determine the language use differences between users who express loneliness compared to a control group of users who did not express loneliness [1]. The word score-based approach was used because prior work [23] used these methods to better understand language features associated with persuasion in online forum posts and comments.

\section{Topic Modeling Approach}

In this section, the natural language processing topic modeling method latent Dirichlet allocation (LDA) [24] was used. LDA works by, first, splitting words in Reddit posts into single words or tokens (tokenization). Second, words that co-occur together are clustered together; the cluster of words are referred to as topics, and based on the content words associated with each topic, a label can be assigned to the topics. For example, LDA could group the words "family," "mom," "dad," "daughter," and "son" as a reference to family. LDA assumes that the topics consist of a combination of words, and each Reddit post is made up of a combination of topics. Using the Dlatk package [25], 20 LDA topics were generated from the /r/AskReddit posts associated with /r/Lonely users and the control group users; to determine the number of LDA topics, the number of topics varied between 5 and 50 topics by starting with 5 topics and incrementing by 2 topics up to 50 topics. A total of 20 topics had the most coherent topic themes when reviewed by the author. With the generated topics, using the Dlatk package [25], the topic themes that frequently occurred in the /r/AskReddit posts by /r/Lonely users when compared with the control group users were identified.

\section{Dictionary-Based Approach}

In this approach, language from /r/AskReddit posts associated with the $/ r /$ Lonely users and the control group users were used to determine the prevalence of Linguistic Inquiry and Word Count (LIWC) [26] dictionary word categories in posts associated with these groups of users. LIWC is a psycholinguistic dictionary made up of 73 predefined categories such as positive and negative emotions; each of these categories has a curated list of words associated with it. LIWC has been used in several prior works $[1,22,27]$. Using the Dlatk package [25], for each group of /r/AskReddit posts (ie, posts belonging to /r/Lonely users and the control group users), the proportion of token words associated with LIWC categories were determined.

\section{Word Score-Based Approach}

The word score-based features valence, arousal, and dominance have been used by prior works to study communication strategies in an online forum [23,28]. Valence indicates the measure of the positive or negative denotation of a word; for example, "enjoyable" is a high valence word and "nightmare" is a low valence word. Arousal measures the emotional intensity expressed in a word; an example of a high arousal word is "exhilarated," and an example of a low arousal word is "siesta." Dominance indicates the measure of the locus of control expressed in a word; for example "powerful" is a high dominance word, and "weak" is a low dominance word. Mohammad [28] provided a lexicon of human ratings for valence, arousal, and dominance for 20,000 words in English; using this lexicon, for each post in the data set, the average ratings of all content words in each of these word categories (ie, valence, arousal, and dominance) was computed.

\section{Results}

\section{Topic Modeling Approach}

Table 2 shows the most significant LDA topics in /r/Askreddit posts by /r/Lonely users compared to the control group.

Table 2. Results from latent Dirichlet allocation analysis on /r/AskReddit posts by /r/Lonely users compared to the control group users.

\begin{tabular}{llll}
\hline Label & Highly correlated words & Cohen $d$ & Mean (SD) \\
\hline Relationships & people, love, hate, relationship, find, can't, lose, meet, stop, married & 0.105 \\
Family and friends/mental health & $\begin{array}{l}\text { family, friends, deal, talk, experience, depression, mental, care, } \\
\text { service, dear }\end{array}$ & 0.10 & 0.038 (0.048) \\
\hline
\end{tabular}

\section{Dictionary-Based Approach}

Table 3 shows the different LIWC categories in /r/AskReddit posts most associated with $/ r /$ Lonely users compared to the control group users.

Table 3. Results from LIWC analysis on /r/AskReddit posts by /r/Lonely users compared to the control group users.

\begin{tabular}{lll}
\hline LIWC $^{\text {a }}$ category & Cohen $d$ & Mean (SD) \\
\hline Social processes & 0.114 & $0.157(0.08)$ \\
Sadness & 0.10 & $0.083(0.042)$ \\
\hline
\end{tabular}

${ }^{\text {a}}$ LIWC: Linguistic Inquiry and Word Count. 


\section{Word Score-Based Approach}

Using Cohen $d$, the effect size between the features that represent the average valence, arousal, and dominance scores for posts in the data set and a feature that represents if a $/ r /$ AskReddit post was by a /r/Lonely user or a control group user was determined, as shown in Table 4.

Table 4. Results from word score-based language feature analysis on /r/AskReddit posts by /r/Lonely users compared to the control group users.

\begin{tabular}{ll}
\hline Attribute & Cohen $d$ \\
\hline Valence & 0.364 \\
Arousal & -0.004 \\
Dominance & 0.117 \\
\hline
\end{tabular}

\section{Discussion}

\section{Findings}

Using natural language processing methods, this study shows the distinction in language use in posts published on a nonloneliness forum by users who express and seek support around loneliness in an online loneliness forum compared to a control group of users. These language use differences reflect the support needs and concerns of these users. The findings from this study are summarized in this section.

This study determined that users who express the feeling of loneliness in $/ r /$ Lonely tend to seek advice and ask questions about relationships on /r/AskReddit (Table 2) compared to the control group users. This finding is in line with prior work [1] that determined that individuals who expressed loneliness on Twitter tend to publish Twitter messages related to themes about difficult interpersonal relationships.

In addition, this study has findings that were not in prior work; specifically, it was observed that individuals who express loneliness in a loneliness forum tend to seek advice and ask questions about mental health concerns as it relates to their family members and friends; for example, the following are examples of $/ r /$ AskReddit posts (rephrased) by /r/Lonely users seeking advice as it relates to their relationships with family members and friends:

\section{I need advice on how to deal with a family member / friend who keeps criticizing me. \\ I need help, if one is struggling with mental health, what is the best way to explain it to family members and friends?}

Using LIWC, it was observed that users who expressed loneliness in the loneliness forum tended to use more words associated with sadness and wanting to socialize in the nonloneliness forum.

Using the word score-based language features valence, arousal, and dominance, it was determined that the average valence and dominance scores in /r/AskReddit posts are more associated with posts by users who express loneliness on $/ r /$ Lonely. A potential explanation for dominance being more associated with /r/AskReddit posts by /r/Lonely users is that some of these users seek support and express vulnerability in these posts; low dominance words suggest vulnerability, hence the association. Additionally, a potential explanation for valence being more associated with $/ r /$ AskReddit posts by $/ r /$ Lonely users is that these users tend to use low valence words in these posts, hence the association.

This study shows that users who express loneliness in a loneliness forum seek support and communicate differently from a control group of users in a nonloneliness forum. The findings from this study can aid in the design and implementation of online loneliness interventions; for example, given that users who express loneliness in the loneliness forum ask questions and seek advice as it relates to their relationships (with family and friends) and use more words associated with seeking to socialize and sadness (Table 3), online loneliness interventions can provide services in which advice and tips are given to users on how to develop, maintain, and navigate relationships.

From this study's findings, when designing and implementing online loneliness interventions, it is important to not only focus on user communication in loneliness forums but also look into how these users communicate in nonloneliness forums.

\section{Limitations}

In this study, data from Reddit users was used and may not be representative of all individuals (some of whom may not publish posts on online forums expressing their feeling of loneliness) who feel lonely.

\section{Ethics and Privacy}

The data set used for this study is publicly available. For all the analyses in this study, no user or moderator of any loneliness forum on Reddit (including $/ r /$ Lonely) was contacted. In addition, besides the usernames of $/ r /$ Lonely users, no other information from user profiles was used or accessed.

\section{Conclusion}

In this study, using natural language processing methods, it was determined that users who express loneliness in an online loneliness forum communicated differently in a nonloneliness forum when compared to a control group of users. The findings from this study can aid with the design and implementation of online loneliness interventions.

\section{Conflicts of Interest}

None declared. 


\section{References}

1. Guntuku SC, Schneider R, Pelullo A, Young J, Wong V, Ungar L, et al. Studying expressions of loneliness in individuals using Twitter: an observational study. BMJ Open 2019 Nov 04;9(11):e030355 [FREE Full text] [doi: 10.1136/bmjopen-2019-030355] [Medline: 31685502]

2. Luhmann M, Hawkley LC. Age differences in loneliness from late adolescence to oldest old age. Dev Psychol 2016 Jun;52(6):943-959 [FREE Full text] [doi: 10.1037/dev0000117] [Medline: 27148782]

3. Beutel ME, Klein EM, Brähler E, Reiner I, Jünger C, Michal M, et al. Loneliness in the general population: prevalence, determinants and relations to mental health. BMC Psychiatry 2017 Mar 20;17(1):97 [FREE Full text] [doi: 10.1186/s12888-017-1262-x] [Medline: 28320380]

4. Newall NEG, Chipperfield JG, Bailis DS, Stewart TL. Consequences of loneliness on physical activity and mortality in older adults and the power of positive emotions. Health Psychol 2013 Aug;32(8):921-924. [doi: 10.1037/a0029413] [Medline: 22888819]

5. Penninx BWJH, van Tilburg T, Kriegsman DMW, Deeg DJH, Boeke AJP, van Eijk JTM. Effects of social support and personal coping resources on mortality in older age: the Longitudinal Aging Study Amsterdam. Am J Epidemiol 1997 Sep 15;146(6):510-519. [doi: 10.1093/oxfordjournals.aje.a009305] [Medline: 9290512]

6. Joiner JT, Rudd MD. Disentangling the interrelations between hopelessness, loneliness, and suicidal ideation. Suicide Life Threat Behav 1996;26(1):19-26. [Medline: 9173606]

7. Wei M, Russell DW, Zakalik RA. Adult attachment, social self-efficacy, self-disclosure, loneliness, and subsequent depression for freshman college students: a longitudinal study. J Counseling Psychol 2005 Oct;52(4):602-614. [doi: 10.1037/0022-0167.52.4.602]

8. Holt-Lunstad J, Smith TB. Loneliness and social isolation as risk factors for CVD: implications for evidence-based patient care and scientific inquiry. Heart 2016 Jul 01;102(13):987-989 [FREE Full text] [doi: 10.1136/heartjnl-2015-309242] [Medline: 27091845]

9. Ruiz C, Ito K, Wakamiya S, Aramaki E. Loneliness in a connected world: analyzing online activity and expressions on real life relationships of lonely users. 2017 Presented at: The AAAI 2017 Spring Symposium on Wellbeing AI: From Machine Learning to Subjectivity Oriented Computing; January 1, 2017; California p. 726-733. [doi: 10.7591/cornell/9781501705335.003.0006]

10. Hunt MG, Marx R, Lipson C, Young J. No more FOMO: limiting social media decreases loneliness and depression. J Soc Clin Psychol 2018 Dec;37(10):751-768. [doi: 10.1521/jscp.2018.37.10.751]

11. Andy A, Guntuku SC, Adusumalli S, Asch DA, Groeneveld PW, Ungar LH, et al. Predicting cardiovascular risk using social media data: performance evaluation of machine-learning models. JMIR Cardio 2021 Feb 19;5(1):e24473 [FREE Full text] [doi: 10.2196/24473] [Medline: 33605888]

12. Andy A, Guntuku S. Does social support expressed in post titles elicit comments in online substance use recovery forums? arXiv. Preprint posted online on November 10, 2020 [FREE Full text]

13. Stokes DC, Andy A, Guntuku SC, Ungar LH, Merchant RM. Public priorities and concerns regarding COVID-19 in an online discussion forum: longitudinal topic modeling. J Gen Intern Med 2020 Jul;35(7):2244-2247 [FREE Full text] [doi: 10.1007/s11606-020-05889-w] [Medline: 32399912]

14. Andy A, Chu B, Fathy R, Bennett B, Stokes D, Guntuku SC. Understanding social support expressed in a COVID-19 online forum. 2021 Apr 01 Presented at: 12th International Workshop on Health Text Mining and Information Analysis; April 2021; Online p. 19-27.

15. Yang D, Kraut R, Levine JM. Commitment of newcomers and old-timers to online health support communities. Proc SIGCHI Conf Hum Factor Comput Syst 2017 May;2017:6363-6375 [FREE Full text] [doi: 10.1145/3025453.3026008] [Medline: 31423492]

16. Yang D, Kraut R, Smith T, Mayfield E, Jurafsky D. Seekers, providers, welcomers, and storytellers: modeling social roles in online health communities. Proc SIGCHI Conf Hum Factor Comput Syst 2019 May;2019:344 [FREE Full text] [doi: 10.1145/3290605.3300574] [Medline: $\underline{31423493]}$

17. Yang D, Yao Z, Seering J, Kraut R. The channel matters: self-disclosure, reciprocity and social support in online cancer support groups. Proc SIGCHI Conf Hum Factor Comput Syst 2019 May;2019:31 [FREE Full text] [doi: 10.1145/3290605.3300261] [Medline: 31448374]

18. Kivran-Swaine F, Ting J, Brubaker J, Teodoro R, Naaman M. Understanding loneliness in social awareness streams: expressions and responses. 2014 May 05 Presented at: Eighth International AAAI Conference on Weblogs and Social Media; June 1-4, 2014; Ann Arbor, MI.

19. Fiesler C, Jiang J, McCann J, Frye K, Brubaker JR. Reddit rules! Characterizing an ecosystem of governance. 2018 Jun 15 Presented at: Twelfth International AAAI Conference on Web and Social Media; June 25-28, 2018; Palo Alto, CA.

20. Fernandes S, Bernardino J. What is BigQuery? In: Proceedings of the 19th International Database Engineering \& Applications Symposium. 2015 Jul Presented at: IDEAS '15; July 13-15, 2015; Yokohama, Japan p. 202-203. [doi: $\underline{10.1145 / 2790755.2790797]}$ 
21. Park G, Yaden DB, Schwartz HA, Kern ML, Eichstaedt JC, Kosinski M, et al. Women are warmer but no less assertive than men: gender and language on Facebook. PLoS One 2016;11(5):e0155885 [FREE Full text] [doi: 10.1371/journal.pone.0155885] [Medline: 27223607]

22. Schwartz HA, Eichstaedt JC, Kern ML, Dziurzynski L, Ramones SM, Agrawal M, et al. Personality, gender, and age in the language of social media: the open-vocabulary approach. PLoS One 2013;8(9):e73791 [FREE Full text] [doi: 10.1371/journal.pone.0073791] [Medline: 24086296]

23. Tan C, Niculae V, Danescu-Niculescu-Mizil C, Lee L. Winning arguments: interaction dynamics and persuasion strategies in good-faith online discussions. In: Proceedings of the 25th International Conference on World Wide Web. 2016 Apr 01 Presented at: WWW '16; April 11-15, 2016; Montréal, QC p. 613-624. [doi: 10.1145/2872427.2883081]

24. Blei DM, Ng AY, Jordan MI. Latent Dirichlet allocation. J Machine Learning Res 2003 Jan 01:993-1022.

25. Schwartz HA, Giorgi S, Sap M, Crutchley P, Ungar L, Eichstaedt J. DLATK: Differential Language Analysis ToolKit. 2017 Jun 05 Presented at: 2017 Conference on Empirical Methods in Natural Language Processing: System Demonstrations; September 2017; Copenhagen, Denmark p. 55-60. [doi: 10.18653/v1/d17-2010]

26. Pennebaker JW, Boyd RL, Jordan K, Blackburn K. The development and psychometric properties of LIWC2015. University Texas Austin 2015 Sep 15:1-10.

27. Guntuku SC, Buffone A, Nanyang KJ, Eichstaedt JC, Ungar LH. Understanding and measuring psychological stress using social media. 2019 May 15 Presented at: Thirteenth International AAAI Conference on Web and Social Media; June 11-14, 2019; Münich, Germany p. 214-225.

28. Mohammad S. Obtaining reliable human ratings of valence, arousal, and dominance for 20,000 English words. 2018 Presented at: 56th Annual Meeting of the Association for Computational Linguistics; July 2018; Melbourne, Australia p. 174-184. [doi: $\underline{10.18653 / \mathrm{v} 1 / \mathrm{p} 18-1017]}$

\section{Abbreviations \\ LDA: latent Dirichlet allocation \\ LIWC: Linguistic Inquiry and Word Count}

Edited by G Eysenbach; submitted 12.03.21; peer-reviewed by C Entwistle, M Fiordelli; comments to author 10.05.21; revised version
received $04.06 .21 ;$ accepted 17.06.21; published 20.07 .21
Please cite as:
Andy A
Studying How Individuals Who Express the Feeling of Loneliness in an Online Loneliness Forum Communicate in a Nonloneliness
Forum: Observational Study
JMIR Form Res $2021 ; 5(7):$ e28738
URL: $\underline{\text { https://formative.jmir.org/2021/7/e } 28738}$
doi: $10.2196 / 28738$
PMID: $\underline{34283026}$

(C)Anietie Andy. Originally published in JMIR Formative Research (https://formative.jmir.org), 20.07.2021. This is an open-access article distributed under the terms of the Creative Commons Attribution License (https://creativecommons.org/licenses/by/4.0/), which permits unrestricted use, distribution, and reproduction in any medium, provided the original work, first published in JMIR Formative Research, is properly cited. The complete bibliographic information, a link to the original publication on https://formative.jmir.org, as well as this copyright and license information must be included. 